\title{
以化学竞赛思维引领的基础化学实验教学改革
}

陶剑波, 胡鸿雨, 谢云龙, 赵国良, 郝仕油 ${ }^{*}$

浙江师范大学行知学院, 浙江 金华 321004

摘要: 以化学竞赛思维为引领, 对两个基础化学实验案例进行教学改革, 拟解决教学改革中遇到的深层次问题。教学改 革结果表明, 相关课程教学目标与专业培养目标能够高度统一; 不同任课教师能在同一教学主题中高度统一思想, 从而 进行深入交流; 通过竞赛思维培养方式, 可极大地激发学生学习动力, 提高他们的学习效率。

关键词: 化学竞赛; 引领; 基础化学实验; 改革

中图分类号: G642; O6

\section{Teaching Reform of Basic Chemistry Laboratory Guided by the Thinking of Chemical Competition}

JianBo Tao, Hongyu Hu, Yunlong Xie, Guoliang Zhao, Shiyou Hao *

Xingzhi College, Zhejiang Normal University, Jinhua 321004, Zhejiang Province, China.

Abstract: Teaching reform of two experiments of basic chemistry laboratory was carried out based on the chemical competition thinking to solve the profound problems in teaching reform. The results of teaching reform show that the teaching objectives of related courses are in good agreement with the training goal of applied chemistry, that the teachers of different courses can highly unify their thoughts under the same teaching topic to communicate deeply, and that training mode of competitive thinking can greatly stimulate students' learning motivation and improve the learning efficiency of students.

Key Words: Chemical competition; Guidance; Basic chemistry laboratory; Reform

众所周知, 化学是一门以实验为基础的古老学科 ${ }^{[1]}$, 因为其理论知识产生于实验, 又不断接受 实验的检验, 从而被世人所接受。因此, 化学是实践论和群众路线的基础。其中基础化学实验在整 个实验体系中尤为重要, 因为它就像房子的地基。基础化学实验课程包含无机、有机、分析、物理 化学实验等, 分别在大一及大二开设, 主要训练学生基本操作及对化学基本原理的理解, 为提高学 生科研素养奠定基础。因此, 如何开设好这些基础实验课程对实现化学人才培养目标至关重要。目 前, 已有相关教师对这些基础实验课程进行了一定程度的改革, 并取得了一定成效, 但随着改革继 续深入, 一些深层次问题逐渐凸显出来。具体如下：1）相关课程教学目标相对独立, 不能协调、统 一地服务于专业总体培养目标, 在现有的教学体系中, 每门课程基本为一个独立的知识体系, 只强 调本课程的知识完整性, 而忽略了前后课程间的相关性, 学生在学习一门课时, 心中只有这本经, 导致站位较低, 不能统领该课程在整个专业中的应有地位; 2) 不同课程任课教师交流不够, 导致课 程间衔接薄弱, 专业体系内逻辑连接性差, 目前, 虽然在课程、课堂、实践等教学环节开展了一些

收稿: 2020-11-17; 录用: 2020-12-07; 网络发表: 2020-12-22

“通讯作者, Email: sky54@zjnu.cn

基金资助：浙江省高等教育十三五第二批教学改革研究项目(jg20190604); 浙江师范大学行知学院教学改革重点项目(xzjg20091201) 
教学改革, 并取得一些成果, 但很难形成专业合力, 导致在申报大型教学项目及申请教学成果奖时, 凸显成果零散、专业特色不突出等问题; 此外, 由于交流不多, 导致专业教师全面发展欠缺; 3) 授 课方式创新性有待提升, 目前, 虽然不同学科教师对相应课程进行了一定的改革与探索, 使教学效 率有了一定的提高, 但学生被动式学习态势并未得到改观, 还是以教师和教材为蓝本, 导致学习效 率和创新性都不高, 且知识综合应用能力极低。

近年来, 各种化学竞赛(如挑战杯、浙江省大学生化学竞赛、全国化工竞赛等)如雨后春笋般涌 现。这些竞赛一般会给出某一研究主题及相关研究背景, 让学生查阅文献、设计实验方案、进行实 验、提交文献综述与研究报告, 从而对参赛学生进行全面评价, 竞赛(特别是决赛阶段)要求学生在原 有基础上推陈出新, 进行创新评价。在这些竞赛过程中, 学生在教师指导下, 对某一主题进行文献 查阅等系列工作, 他们的主动性与积极性能够充分被调动; 通过与教师交流、讨论相关问题, 学生 在创新及知识综合应用方面也受益罒浅。

基于以上分析, 我们提出了 “以化学竞赛思维引领的基础化学实验教学改革” 理念, 拟解决上 述在教学改革中出现的深层次问题。本文通过对两个经典实验案例的教学改革, 阐述化学竞赛思维 在基础化学实验教学中的引领作用。

\section{1 经典实验教学案例}

\section{1 碱式碳酸铜 $\left(\mathrm{Cu}_{2}(\mathrm{OH})_{2} \mathrm{CO}_{3}\right)$ 合成条件探索}

碱式碳酸铜为孔雀绿细小无定型粉末, 不溶于水和醇, 而溶于酸、氨水及氰化钾溶液。通常采 用 $\mathrm{CuSO}_{4}$ 和 $\mathrm{Na}_{2} \mathrm{CO}_{3}\left(\mathrm{NaHCO}_{3}\right)$ 为原料合成 $\mathrm{Cu}_{2}(\mathrm{OH})_{2} \mathrm{CO}_{3}$, 以产物颜色是否为孔雀绿为判断标准。大多 数师范院校采用北京师范大学无机化学教研室等合编的教材, 它是以 $\mathrm{CuSO}_{4}$ 和 $\mathrm{Na}_{2} \mathrm{CO}_{3}$ 为原料合成 $\mathrm{Cu}_{2}(\mathrm{OH})_{2} \mathrm{CO}_{3}{ }^{[2]}$, 反应方程式如下:

$$
2 \mathrm{CuSO}_{4}+2 \mathrm{Na}_{2} \mathrm{CO}_{3}+\mathrm{H}_{2} \mathrm{O}=\mathrm{Cu}_{2}(\mathrm{OH})_{2} \mathrm{CO}_{3}+2 \mathrm{Na}_{2} \mathrm{SO}_{4}+\mathrm{CO}_{2}
$$

在上述反应体系中沉淀产物主要为 $\mathrm{Cu}_{2}(\mathrm{OH})_{2} \mathrm{CO}_{3}$, 若反应条件控制不好, 则可能产生 $\mathrm{Cu}(\mathrm{OH})_{2}$ 和 $\mathrm{CuCO}_{3}$, 因此, 能否获得高纯度的 $\mathrm{Cu}_{2}(\mathrm{OH})_{2} \mathrm{CO}_{3}$ 与反应条件密切相关。据理论分析可知, 影响 $\mathrm{Cu}_{2}(\mathrm{OH})_{2} \mathrm{CO}_{3}$ 性质的实验因素有反应原料配比、反应体系温度、反应体系 $\mathrm{pH}$ 、反应陈化时间等。在 北师大等高校合编的教材中, 主要考查 $\mathrm{CuSO}_{4}$ 和 $\mathrm{Na}_{2} \mathrm{CO}_{3}$ 的配比及反应体系温度对产物性质的影响。

实验具体过程如下:

首先配制 $0.5 \mathrm{~mol} \cdot \mathrm{L}^{-1}$ 的 $\mathrm{CuSO}_{4}$ 和 $\mathrm{Na}_{2} \mathrm{CO}_{3}$ 溶液各 $100 \mathrm{~mL}$, 室温下按体积比为 $1.0: 1.0 、 1.2: 1.0$ 、 $0.8: 1.0$ 的比例混合 $\mathrm{CuSO}_{4}$ 和 $\mathrm{Na}_{2} \mathrm{CO}_{3}$ 溶液, 选出沉淀产物颜色接近孔雀绿的反应体系; 再配制该比例 的 $\mathrm{CuSO}_{4}$ 和 $\mathrm{Na}_{2} \mathrm{CO}_{3}$ 溶液, 在 $60 、 80 、 100^{\circ} \mathrm{C}$ 的水浴中加热一定时间, 再通过颜色对比, 确定适宜的反 应温度; 最后放大合成产生相应产物。

实验结果表明, 很多学生获得了蓝色产物, 有些学生获得的产物甚至部分发黑。到底是什么原 因导致该现象的发生呢? 以往由于实验课堂时间有限, 学生只能发现问题, 不能理解深层次原因。 有的学生也只是问问实验教师相关原因, 然后把相关现象、结果与可能原因写到实验报告中, 并未 进行深入分析和跟进实验, 所以不了了之, 导致学生探索学习的主动性严重受阻。自从我们引进 “以 化学竞赛思维引领的基础化学实验教学改革” 理念后, 对该实验项目进行了跟进实验, 特意另设一 门实验课程: 探索式无机合成实验, 该课程利用6课时对 $\mathrm{Cu}_{2}(\mathrm{OH})_{2} \mathrm{CO}_{3}$ 合成的影响因素进行探究。

课前, 教师利用一定时间对前期实验中遇到的问题进行导向性提示, 要求学生回去进行文献检 索, 让学生弄明白不成功实验中生成的蓝色和黑色物质是什么, 是什么原因导致的, 如何解决这些 问题。要求学生 4-6人组成学习小组进行文献查阅与讨论, 并写出检索报告, 发给老师。老师根据学 生写的检索报告, 进行总结, 再把总结返还给学生, 让他们明白蓝色物质主要是 $\mathrm{Cu}(\mathrm{OH})_{2}$, 黑色物质 主要是 $\mathrm{CuO} 。 \mathrm{Cu}(\mathrm{OH})_{2}$ 产生的原因是溶液中 $\mathrm{Cu}^{2+}$ 浓度与 $\mathrm{OH}^{-}$浓度平方之积大于 $\mathrm{Cu}(\mathrm{OH})_{2}$ 的 $K_{\mathrm{sp}}$, 其中 $\mathrm{Cu}^{2+}$ 来源于 $\mathrm{CuSO}_{4}$ 在水中的电离, $\mathrm{OH}^{-}$产生于 $\mathrm{CO}_{3}^{2-}$ 的水解; $\mathrm{Cu}^{2+}$ 浓度大小可通过控制 $\mathrm{CuSO}_{4}$ 的初始浓度实 
现, 那么 $\mathrm{OH}^{-}$浓度是由什么因素控制呢? 要求学生根据无机化学水解相关知识及物理化学热力学相 关知识进行解答, 不懂之处可请教无机化学及物理化学教师。 $\mathrm{CuO}$ 可能是 $\mathrm{Cu}_{2}(\mathrm{OH})_{2} \mathrm{CO}_{3}$ 热分解产生, 也可能是 $\mathrm{Cu}(\mathrm{OH})_{2}$ 热分解产生, 要求学生通过物理化学热力学相关知识寻求答案。基于以上分析, 再 次要求学生查阅文献进行深入分析。根据文献查阅可知 ${ }^{[3,4]}$, 当反应体系中 $\mathrm{OH}^{-}$浓度较高时, 易生成 蓝色 $\mathrm{Cu}(\mathrm{OH})_{2}$ 沉淀, 且易分解; 当 $\mathrm{OH}^{-}$浓度较低时, 易生成绿色的碱式铜盐, 且较稳定。据此, 学生 再次设计方案进行实验。实验结果表明, 影响 $\mathrm{Cu}_{2}(\mathrm{OH})_{2} \mathrm{CO}_{3}$ 合成的主要实验因素有 $\mathrm{CuSO}_{4}$ 和 $\mathrm{Na}_{2} \mathrm{CO}_{3}$ 的 初始浓度和反应体系温度, 当 $\mathrm{CuSO}_{4}$ 与 $\mathrm{Na}_{2} \mathrm{CO}_{3}$ 的物质的量之比为 $1: 1$ 左右, 反应温度为 $75-85^{\circ} \mathrm{C}$ 时, 所合成的产物颜色为浅绿色, 因为此时 $\mathrm{OH}^{-}$浓度较低, 不足以完全沉淀溶液中的 $\mathrm{Cu}^{2+}$, 需要结合部分 $\mathrm{CO}_{3}^{2-}$ 生成 $\mathrm{Cu}_{2}(\mathrm{OH})_{2} \mathrm{CO}_{3}$ 。为了确定 $\mathrm{Cu}_{2}(\mathrm{OH})_{2} \mathrm{CO}_{3}$ 的成分, 再次要求学生在分析化学教师的指导下, 设 计相关实验方案进行实验。实验结果表明, 所合成的产物与 $\mathrm{Cu}_{2}(\mathrm{OH})_{2} \mathrm{CO}_{3}$ 理论值有一定的出入; 此时 要求学生进一步进行文献检索并分析, 寻求其他可能影响产物性能的因素。通过检索发现, 影响 $\mathrm{CO}_{3}^{2-}$ 水解的因素除了温度之外, 还有反应体系的 $\mathrm{pH}{ }^{[5]} ; \mathrm{Cu}_{2}(\mathrm{OH})_{2} \mathrm{CO}_{3}$ 不稳定的因素除反应体系温度外, 还有陈化时间 ${ }^{[6]}$ 。因此, 基于以上分析结果, 再次让学生设计新的实验方案并进行实验, 确定 $\mathrm{CuSO}_{4}$ 与 $\mathrm{Na}_{2} \mathrm{CO}_{3}$ 的物质的量之比为 $1:$ 左右, 反应体系温度在 $75-85{ }^{\circ} \mathrm{C}, \mathrm{pH}$ 在 7.5-8.5 (利用 $0.1 \mathrm{~mol} \cdot \mathrm{L}^{-1}$ 的 $\mathrm{NaOH}$ 溶液调节), 陈化时间在20-40 min, 所合成的产物颜色为浅绿色, 且其组成与 $\mathrm{Cu}_{2}(\mathrm{OH})_{2} \mathrm{CO}_{3}$ 的 理论值接近, 证明探索的实验条件适宜合成目标产物。实验进行到此, 似乎可以画上句号。然而有 部分学生抱怨很累, 觉得实验周期长, 因为要逐个考查不同因素的不同值。

针对该问题, 指导教师对学生进行导向性提问: 能否找到一种方法既能减少实验工作量, 又能 较全面地考查各实验因素的不同值? 要求他们再次进行文献查阅, 寻求相关方案。结果有学生找到 了正交实验法, 认为该方法可满足要求。因此, 教师再利用一定时间给学生讲解正交实验法的原理 和设计方案种类, 并发给学生相关资料, 让他们自行设计正交实验表格。在综合前期实验基础上, 学生设计出了4因素3水平正交表(详见表1), 并进行相关实验, 实验结果表明, 5 号实验结果较理想, 不仅颜色为浅绿色, 且其成分与 $\mathrm{Cu}_{2}(\mathrm{OH})_{2} \mathrm{CO}_{3}$ 理论值相近, 热稳定性较好: 在 $80{ }^{\circ} \mathrm{C}$ 下可稳定较长时 间。

表1 正交实验表 $\left[\mathbf{L} 9\left(3^{4}\right)\right]$

\begin{tabular}{ccccc}
\hline 实验 & $n_{\mathrm{CuSO}_{4}:}: n_{\mathrm{NaCO}_{3}}$ & 温度 $/{ }^{\circ} \mathrm{C}$ & $\mathrm{pH}$ & 陈化时间 $/ \mathrm{min}$ \\
\hline 1 & $0.8: 1.0$ & 60 & 6.5 & 20 \\
2 & $0.8: 1.0$ & 80 & 7.5 & 40 \\
3 & $0.8: 1.0$ & 100 & 8.5 & 60 \\
4 & $1.0: 1.0$ & 60 & 7.5 & 60 \\
5 & $1.0: 1.0$ & 80 & 8.5 & 20 \\
6 & $1.0: 1.0$ & 100 & 6.5 & 40 \\
7 & $1.2: 1.0$ & 60 & 8.5 & 40 \\
8 & $1.2: 1.0$ & 80 & 6.5 & 60 \\
9 & $1.2: 1.0$ & 100 & 7.5 & 20 \\
\hline
\end{tabular}

\section{2 复方氢氧化铝药片中铝含量测定影响因素的探索}

复方氢氧化铝药片是一种抗胃酸药, 其主要成分是氢氧化铝、三硅酸镁及少量颠茄流浸膏, 其 中铝和镁的含量可用EDTA为滴定剂进行测定 ${ }^{[6]}$ 。依据 $\mathrm{Al}^{3+}$ 与EDTA能形成稳定的 $1: 1$ 络合物的性 质, 用二甲酚橙作指示剂测定铝。考虑到 $\mathrm{Al}^{3+}$ 与EDTA的反应比较慢且对二甲酚橙指示剂有封闭作用, 故采用返滴定法 ${ }^{[7]}$ 。 
要想获得理想的实验结果, 必须了解影响滴定结果的因素。那么如何确定这些因素呢? 由于课 堂时间有限, 课前指导教师把学生按 4 人一组进行分组, 让学生带着问题(影响滴定结果的相关因素) 去查找文献资料, 并认真做好实验预习报告。教师根据学生的预习报告进行总结并反馈给学生。让 学生知道本实验中 $\mathrm{Al}^{3+}$ 容易发生水解, 为了防止水解, 必须调节滴定体系的 $\mathrm{pH}$, 以使结果准确。学 生通过反复实验, 并经各小组交流总结, 得出滴定体系 $\mathrm{pH}$ 在3-4最好, 与相关文献结果一致 ${ }^{[7]}$ 。具体 操作如下: 首先在试液中滴加二甲酚橙指示剂至溶液呈黄色, 然后滴加体积比为 $1: 1$ 的氨水(1体积 质量百分比为 $28 \%$ 的氨水与 1 体积水的混合液)至溶液呈紫红色, 再滴加体积比为 $1: 3$ 的 HCl溶液( 1 体 积质量百分比为 $37 \%$ 的盐酸与 3 体积水的混合液)使滴定体系刚好显黄色, 再用体积比为 $1: 3$ 的 HCl溶 液调节其 $\mathrm{pH}=3-4$ 。

尽管滴定体系酸度确定后, 可使结果较准确, 还是有不少学生获得的实验结果比理论值低。为 此, 指导教师要求学生再次查找文献资料并分析, 寻找其他可能影响实验结果的因素。通过检索发 现, EDTA标准溶液加入量也有重要影响: 若加入量过少, 则不能完全与 $\mathrm{Al}^{3+}$ 发生络合反应, 测定结 果将偏小; 若加入量过多, 使得返滴定的锌标准溶液需要量增大, 导致系统误差变大。因此, 学生 根据分析结果进行实验, 获得较理想的EDTA用量。此外, 通过检索发现 $\mathrm{Al}^{3+}$ 与EDTA的络合速度也 会影响实验结果, 通常采用加热至沸加速反应, 以使实验结果接近理论值。此时, 有些同学提出, 他们当时实验结果出现偏差, 是因为溶液加热还未沸腾就开始下一步实验, 导致 $\mathrm{Al}^{3+}$ 与EDTA络合不 完全。基于以上实验结果, 再次进行实验, 学生确定以下实验过程: 先将一定量 $(m$ 克)的药片研细, 逐滴加入体积比为 $1: 1$ 的 $\mathrm{HCl}$ 溶液 (1体积质量百分比为 $37 \%$ 的盐酸与 1 体积水的混合液) 以使药片溶 解, 再加纯水并加热煮沸, 静置冷却后, 抽滤、定容至 $250 \mathrm{~mL}$; 取一定量试液 $V_{1} \mathrm{~mL}$, 加入定量且过 量的EDTA标准溶液 $V_{2} \mathrm{~mL}$, 滴加二甲酚橙指示剂至溶液呈黄色, 然后滴加体积比为 $1: 1$ 的氨水, 使 溶液为紫红色, 再滴加体积比为 $1: 3$ 的 $\mathrm{HCl}$ 使溶液显黄色, 且用体积比为 $1: 3$ 的 $\mathrm{HCl}$ 溶液调节滴定体 系 $\mathrm{pH}$ 为3-4; 加热至沸并保持 $3 \mathrm{~min}$, 冷却至室温后, 加入质量百分比为 $20 \%$ 的六亚甲基四胺, 此时 溶液显黄色, $\mathrm{pH}=5-6$; 补加二甲酚橙指示剂, 用锌标准溶液返滴定剩余的EDTA标准溶液, 溶液由 亮黄色突变为紫红色即为滴定终点。根据下列公式计算铝的质量含量 $(w \%)$ 。

$$
w \%=\frac{0.25 C_{\mathrm{EDTA}}\left(V_{2}-V_{3}\right) M_{\mathrm{Al}(\mathrm{OH})_{3}}}{m V_{1}}
$$

式中 $C_{\mathrm{EDTA}}$ 为 $\mathrm{EDTA}$ 的浓度 $\left(\mathrm{mol} \cdot \mathrm{L}^{-1}\right), V_{3}$ 为锌标准溶液用量 $(\mathrm{mL}), M_{\mathrm{Al}(\mathrm{OH})_{3}}$ 为 $\mathrm{Al}(\mathrm{OH})_{3}$ 的摩尔质量, 计算 时式中体积单位全部转换成 $\mathrm{L}$ 。

此时, 实验好像结束, 但部分学生在查找文献资料时, 发现其他方法也可测定复方氢氧化铝药 片中铝含量。因此, 指导教师就让这些感兴趣的学生再次组队讨论, 根据文献整理的滴定方法如下: 以PAN (1-(2-吡啶偶氮)-2-菜酚)为指示剂, 用铜标准溶液返滴定剩余的EDTA标准溶液, 溶液由黄色转 变为绿色再突变为紫色, 即为终点 ${ }^{[8]}$ 。此外, 有的学生检索到测定复方氢氧化铝片中镁含量的方法, 文献资料显示用直接滴定法测定复方氢氧化铝片中镁含量较好。根据文献, 学生自行组队进行讨论, 确定实验方案如下: 滴定前, 通过调节溶液 $\mathrm{pH}$, 使体系中 $\mathrm{Al}^{3+}$ 沉淀, 过滤获得滤液; 在滤液中加入 一定量的三乙醇胺, 隐蔽滤液中少量的 $\mathrm{Al}^{3+}$, 再加氨缓冲溶液及铬黑 $\mathrm{T}$ 指示剂; 最后用EDTA标准溶 液滴定使溶液从酒红色突变成蓝色 ${ }^{[9]}$, 即为滴定终点。

\section{2 教学总结与反思}

以化学竞赛思维为引领, 对两个经典实验教学案例进行改革, 获得以下教学成效: 第一、课程 虽不同, 但目标一致, 在改革指导思维指导下, 可使相关课程教学目标高度统一到专业培养目标上; 第二、在同一实验教学案例中, 涉及无机、分析、物化及有机等内容, 因此, 可使不同课程教师围 绕同一教学主题高度统一思想, 从而进行深入交流, 为更好地实现专业培养目标奠定基础; 第三、 通过化学竞赛培养方式, 可激发学生学习动力, 变被动学习为主动学习, 从而提高学习效率。如何 
才能使得教学改革效果更好呢? 我们认为, 首先要解决好教学改革过程中遇到的问题: 主要是教师 跟进不到位所导致的学生学习动力下降、精神涣散等问题, 这需要学校从制度方面进行改革, 如对 参与教学改革的教师进行一定程度的激励; 其次是实验场所问题, 学生反复实验需要场所, 有时还 需要在夜间开放实验室, 但会遇到人员短缺及实验室冲突等问题, 导致学生想要开展的实验进行不 了, 因而影响教学改革效果及学生学习动力, 同样也需要学校从制度上加以保障。通过实验案例二 可知, 为了更好地提高改革效率, 充分调动学生的聪明才智也是非常重要的举措。本来以为在教师 指导下通过一种方法可完成复方氢氧化铝片中铝含量的测定, 但在学生的努力下, 改善并提出了第 二种实验方案, 还提出了镁的滴定方案, 这为完善整个滴定实验奠定了坚实基础, 此外也极大地提 高了学生的科研热情。

\section{参 考 文 献}

[1] 赵新华. 无机化学实验. 第4版. 北京: 高等教育出版社, 2014.

[2] 北京师范大学无机化学教研室. 无机化学实验. 第4版. 北京: 高等教育出版社, 2007.

[3] 刘怀乐. 化学教育, 2004, 26 (10), 53.

[4] 孙跃枝. 化学工程与装备, 2009, 38 (2), 27.

[5] 大连理工大学无机化学教研室. 无机化学. 第6版. 北京: 高等教育出版社, 2018.

[6] 王术皓. 分析化学实验. 青岛: 中国海洋大学出版社, 2009.

[7] 张国功, 苑壮东, 孔亚杰, 王静霞, 崔南南. 济宁学院学报, 2015, 36 (3), 51.

[8] 徐伟亮. 基础化学实验. 第2版. 北京: 科学出版社, 2010.

[9] 蔡维平. 基础化学实验 (一). 厦门: 科学出版社, 2010. 